\title{
Roles de la resolución de problemas en el currículo oficial
}

\author{
Daniela Olivares, Universidad de Granada (España) \\ Isidoro Segovia, Universidad de Granada (España) \\ José Luis Lupiáñez, Universidad de Granada (España)
}

\section{Roles de la resolución de problemas en el currículo oficial}

\section{Resumen}

En este trabajo describimos el papel de la resolución de problemas en distintos documentos que componen el currículo oficial de educación primaria en Chile. Nuestro marco teórico toma en cuenta tres dimensiones de la resolución de problemas: los roles que puede tomar según Schoenfeld (1992) y Schroeder y Lester (1989), las características que facilitan su incorporación al currículo y los tipos de problemas sugeridos en los documentos curriculares. Elaboramos un sistema de categorías y llevamos a cabo un análisis de contenido. Concluimos que los documentos reflejan visiones diferentes y hasta contradictorias sobre el papel que juega la resolución de problemas para la enseñanza de las matemáticas, dificultando su total implementación según lo dispuesto en la normativa oficial.

Palabras clave. Resolución de problemas; currículo de matemáticas; normativas curriculares; tipos de problemas; análisis de contenido

\section{Roles of problem solving in the official curriculum}

\section{Abstract}

In this work we describe the role of problem solving in different documents of the Chilean official curriculum in primary education. Our theoretical framework takes into account three dimensions of problem solving: the roles it can play according to Schoenfeld (1992), and Schroeder and Lester (1989); the characteristics that facilitate its incorporation into the curriculum and the types of problems suggested in the curricular documents. We developed a system of categories and carried out a content analysis. We conclude that the documents reflect different and even contradictory views on the role of problem solving for the teaching of mathematics, hindering its full implementation as provided in official regulations.

Keywords. Problem solving; mathematics curriculum; curricular regulations; types of problems; content analysis

\section{Introducción}

Mucho se ha avanzado en Didáctica de la Matemática sobre resolución de problemas. Mientras que hay una larga tradición de investigación, que se concreta en un conjunto importante de trabajos que aún no se ha terminado de sistematizar (Castro, 2008), en el ámbito del aula el panorama parece ser distinto. A pesar de que en muchas propuestas curriculares se pretende dar un rol protagónico a la resolución de problemas, esto no se ve reflejado en las prácticas de enseñanza (Burkhardt, 2014). ¿Qué puede ocurrir para que exista esta brecha entre las intenciones de los diseñadores curriculares y la realidad? Choppin, McDuffie, Drake y Davis (2018) señalan que un factor importante son las interpretaciones de los profesores sobre lo planteado en el currículo. Por otro lado, Burkhardt (2014) indica que cuando, en documentos diversos, se transmiten mensajes diferentes acerca de lo que se espera, se obtienen resultados que pueden ser contrarios a las intenciones originales. Esto lleva a preguntarnos: ¿qué mensaje se está transmitiendo sobre la resolución de problemas? y, ¿qué papel juega la resolución de problemas en los distintos documentos curriculares? El objetivo de este trabajo es describir el papel de la resolución de problemas en distintos 
documentos del currículo oficial de educación primaria en Chile, en concreto, del cuarto curso de educación primaria. La elección de este sistema educativo se justifica por la extensa variedad de documentos normativos y materiales de apoyo que ofrece, pero que sin embargo demuestra altos niveles de desigualdad educativa (Oliva, 2017).

Nuestros objetivos específicos son: (1) Determinar qué rol de la resolución de problemas con respecto a la enseñanza de las matemáticas se promueve en los distintos documentos curriculares. (2) Identificar qué características metodológicas, sugeridas por la literatura para el trabajo con la resolución de problemas, se están considerando en estos documentos. (3) Caracterizar los tipos de problemas sugeridos por el currículo.

\section{Marco teórico}

En nuestro marco teórico, primero establecemos qué entenderemos por currículo de matemáticas y sus niveles de diseño e implementación, y luego nos centramos en la resolución de problemas y su papel en la enseñanza de las matemáticas.

\subsection{El currículo de matemáticas}

El currículo es un concepto que puede tener muchos significados, por lo que es necesario aclarar a qué nos estamos refiriendo. En nuestro caso adoptamos la propuesta de Remillard y Heck (2014), quienes definen el currículo de matemáticas como un plan de las experiencias que los estudiantes encontrarán, así como aquellas experiencias que, de hecho encuentran, y que han sido diseñadas para ayudarlos a alcanzar objetivos matemáticos específicos. Estos autores proponen un modelo de implementación curricular, formado por varios niveles, uno de los cuales corresponde al currículo oficial. Este está compuesto por objetivos (expresados en las normativas oficiales), materiales que apoyan su implementación y el contenido de evaluaciones oficiales, por lo general de carácter nacional y que suelen traer consecuencias para profesores y estudiantes. La visión de estos autores coincide con la de Rico y Moreno (2016), quienes señalan que el currículo de matemáticas corresponde a una propuesta formativa, situada entre lo prescrito y lo implementado en el aula. Documentos como los del currículo oficial pueden apoyar la toma de decisiones que el profesor lleva a cabo en el aula, siempre que ellos estén alineados en una visión común y den espacio para realizar adaptaciones productivas (Burkhardt, 2014; Choppin et. al, 2018).

\subsection{El papel de la resolución de problemas en la enseñanza de matemáticas}

Entendemos el concepto de problema como una tarea que no puede ser resuelta mediante un esfuerzo directo, sino que su método de solución no se conoce de antemano. Resolver un problema depende de quien se enfrente a él, ya que, dependiendo de los conocimientos del resolutor, la tarea puede o no resultarle desafiante, matemáticamente rica, o exigirle procesos de razonamiento (Burkhardt, 2014; Schoenfeld, 1985).

La resolución de problemas en la enseñanza puede adoptar distintos roles. Para identificarlos, Schoenfeld (1992) usa las acepciones del Diccionario Webster (ver https://www.merriam-webster.com) para el término problema. La primera dice que en matemáticas, es cualquier cosa que requiere hacer algo. La segunda define a un problema como una pregunta desconcertante, de modo que se trataría de la esencia de la matemática. Schoenfeld señala que la primera acepción es la que se suele encontrar a nivel escolar: se enseñan contenidos, procedimientos y finalmente se presentan problemas para ejercitar lo aprendido. Otra clasificación es la de Schroeder y Lester (1989). Según estos autores, en matemáticas se puede enseñar 'sobre' la resolución de 
problemas, cuando se aborda como habilidad y como contenido de enseñanza en sí mismo, se promueven las etapas de Polya y se instruye en el uso de heurísticas. También se puede enseñar 'para' resolver problemas, cuando se enseñan primero contenidos y procedimientos para luego enfrentarse a problemas. Estas dos corresponderían a la primera acepción de Schoenfeld. Schroeder y Lester (1989) además señalan la enseñanza 'a través' de la resolución de problemas, descubriendo el contenido a través de problemas no rutinarios, lo que coincide con la segunda acepción de Schoenfeld.

Estos roles se ven acompañados por características que puede adquirir la resolución de problemas cuando se incorpora al currículo. Por ejemplo, en la literatura se promueve el uso de problemas como medio para enseñar y aprender nuevos contenidos, así como para la evaluación de los aprendizajes (Stacey, 2005). Otro aspecto importante es la ubicación temporal de los problemas para alcanzar ciertos objetivos instruccionales. Un problema al inicio de una secuencia didáctica puede usarse para descubrir una idea matemática, pero el mismo presentado después de la enseñanza de un procedimiento se puede convertir en ejercicio de aplicación (Leong et al., 2016). Las orientaciones que entregan los documentos curriculares a los profesores también resultan valiosas a la hora de implementar la resolución de problemas ya que es parte del conocimiento que estos deben manejar. Entre los aspectos a incluir se encuentran: cómo evaluar el progreso de los estudiantes en el ámbito de la resolución de problemas, la naturaleza de los problemas matemáticos, orientaciones sobre cómo trabajarlos y cómo abordar la invención de problemas (Piñeiro, Castro-Rodriguez y Castro, 2016; Stacey, 2005).

Por último, el papel de la resolución de problemas se puede ver reforzado por los tipos de problemas sugeridos en los documentos curriculares. Zhu y Fan (2006) presentan una clasificación de tipos que se pueden encontrar en los libros de texto: problemas rutinarios frente a no rutinarios; de final abierto y de final cerrado; de uno o varios pasos para su resolución (Castro, 1994, los denomina 'simples' o 'compuestos'); con datos suficientes, insuficientes o superfluos; de aplicación o no a la vida real. En este último caso, la clasificación de PISA considera problemas que abordan situaciones personales, públicas, laborales o educativas y científicas (OECD, 2013).

\section{Método}

A continuación, damos a conocer el método de este estudio, el cual tuvo un enfoque cualitativo-interpretativo. Comenzaremos dando a conocer la muestra analizada, para luego exponer en detalle el método de análisis de datos.

\subsection{Muestra}

Tomamos el caso del currículo chileno por contar con una amplia variedad de documentos diseñados y distribuidos para apoyar su implementación en el aula. En su conjunto, estos materiales prescriben la forma en que ha de llevarse a cabo la enseñanza. Los documentos se seleccionaron en función de los siguientes criterios: ser elaborados por el Ministerio de Educación, tener relación con aspectos curriculares, y estar a disposición pública. La Tabla 1 muestra los documentos curriculares (DC) analizados.

Tabla 1. Documentos curriculares analizados

\begin{tabular}{|c|c|c|c|}
\hline Documento & Código & Referencia & Finalidad \\
\hline Ley General de Educación & DC1 & $\begin{array}{c}\text { Ley N } \\
20.370,2009\end{array}$ & $\begin{array}{c}\text { Regular el sistema educativo y la } \\
\text { enseñanza a nivel escolar }\end{array}$ \\
\hline Bases Curriculares & DC2 & $\begin{array}{c}\text { MINEDUC, } \\
\text { 2012a }\end{array}$ & $\begin{array}{c}\text { Documento principal que norma } \\
\text { el currículo }\end{array}$ \\
\hline
\end{tabular}




\begin{tabular}{|c|c|c|c|}
\hline Programa de Estudio & DC3 & $\begin{array}{c}\text { MNEDUC, } \\
\text { 2012b }\end{array}$ & $\begin{array}{c}\text { Organizar temporalmente los } \\
\text { objetivos de las Bases } \\
\text { Curriculares, y entregar } \\
\text { sugerencias metodológicas y de } \\
\text { evaluación }\end{array}$ \\
\hline $\begin{array}{c}\text { Material Pedagógico para el } \\
\text { Aula }\end{array}$ & DC4 & $\begin{array}{c}\text { MINEDUC, } \\
\text { 2012c }\end{array}$ & $\begin{array}{c}\text { Proporcionar material didáctico } \\
\text { de apoyo }\end{array}$ \\
\hline $\begin{array}{c}\text { Bases Administrativas y } \\
\text { Técnicas para la Licitación } \\
\text { de Textos Escolares }\end{array}$ & DC5 & $\begin{array}{c}\text { MINEDUC, } \\
2017\end{array}$ & $\begin{array}{c}\text { Entregar lineamientos a las } \\
\text { editoriales de textos escolares }\end{array}$ \\
\hline $\begin{array}{c}\text { Estándares de Aprendizaje } \\
\text { MINEDUC, } \\
2013\end{array}$ & DC6 & $\begin{array}{c}\text { Interpretar los resultados de la } \\
\text { prueba nacional SIMCE }\end{array}$ \\
\hline $\begin{array}{c}\text { Estándares Orientadores } \\
\text { para Egresados de Carreras } \\
\text { de Pedagogía }\end{array}$ & DC7 & $\begin{array}{c}\text { MINEDUC, } \\
2011\end{array}$ & $\begin{array}{c}\text { Servir de referencia para la } \\
\text { formación de profesores de } \\
\text { educación primaria }\end{array}$ \\
\hline
\end{tabular}

De los documentos señalados consideramos una muestra centrada en el cuarto curso de educación primaria. Este curso resulta de interés en el contexto chileno ya que corresponde la aplicación de la prueba nacional SIMCE (Sistema de Medición de la Calidad de la Educación), que influye fuertemente en el currículo (Ruminot, 2017).

\subsection{Método de análisis de datos}

Llevamos a cabo un análisis de contenido, en el cual participó el equipo investigador junto con dos colaboradores que ayudaron en la codificación. Primero establecimos un sistema de categorías a priori, con tres dimensiones de acuerdo a los objetivos específicos: roles de la resolución de problemas en la enseñanza de la matemática, características de la incorporación de la resolución de problemas al currículo y tipos de problemas matemáticos. Mediante una revisión de la literatura, determinamos las categorías y sub-categorías constituyentes de cada dimensión. La organización en categorías y sub-categorías fue dada por la literatura, así como por su nivel de concreción. La Tabla 2 presenta el desglose de la Dimensión 1 para posibles roles de la resolución de problemas según Schoenfeld (1992) y Schroeder y Lester (1989).

Tabla 2. Dimensión 1: Roles de la resolución de problemas en la enseñanza de las matemáticas

\begin{tabular}{|c|c|c|}
\hline Categoría & Sub-categoría & Descripción \\
\hline Concebir el problema & $\begin{array}{c}\text { Enseñar para resolver } \\
\text { problemas } \\
\text { como cualquier cosa } \\
\text { que requiere ser hecha }\end{array}$ & $\begin{array}{c}\text { Primero se enseña contenido regular para } \\
\text { luego resolver problemas. Éstos se utilizan } \\
\text { como contexto para algo más, e.g. } \\
\text { practicar procedimientos. }\end{array}$ \\
\cline { 2 - 3 } & $\begin{array}{c}\text { Enseñar sobre la } \\
\text { resolución de problemas }\end{array}$ & $\begin{array}{c}\text { Aparece en el currículo como una } \\
\text { habilidad a enseñar. Implica resolver } \\
\text { problemas no rutinarios después de } \\
\text { problemas rutinarios. Se enseñan } \\
\text { estrategias y heurísticas como las } \\
\text { sugeridas por Pólya. }\end{array}$ \\
\hline $\begin{array}{c}\text { Concebir el problema } \\
\text { como una pregunta } \\
\text { desconcertante }\end{array}$ & $\begin{array}{c}\text { Enseñar a través de la } \\
\text { resolución de problemas }\end{array}$ & $\begin{array}{c}\text { Se enseña el contenido del currículo a } \\
\text { través de problemas no rutinarios que } \\
\text { aparecen regularmente. El conocimiento } \\
\text { surge a medida que se solucionan } \\
\text { problemas como método de aprendizaje. }\end{array}$ \\
\hline
\end{tabular}


La Tabla 3 muestra las categorías y sub-categorías de la Dimensión 2 sobre características de la incorporación de la resolución de problemas al currículo. Para esta dimensión consideramos principalmente el sistema de categorías de Piñeiro et al. (2016), así como los aportes de Leong et al. (2016), Lester y Cai (2016) y Stacey (2005).

Tabla 3. Dimensión 2: Características de la incorporación de la resolución de problemas

\begin{tabular}{|c|c|c|}
\hline Categoría & Sub-categoría & Descripción \\
\hline \multirow{3}{*}{$\begin{array}{l}\text { Evidencias de } \\
\text { imbricación en } \\
\text { el currículo }\end{array}$} & Imbricación en la enseñanza & $\begin{array}{l}\text { La resolución de problemas aparece apoyando } \\
\text { al currículo normal en la mayor parte de éste. }\end{array}$ \\
\hline & Imbricación en el aprendizaje & $\begin{array}{c}\text { Se promueve que los estudiantes aprendan } \\
\text { nuevos conocimientos resolviendo problemas } \\
\text { como actividad principal. }\end{array}$ \\
\hline & Imbricación en la evaluación & $\begin{array}{l}\text { La evaluación de la resolución de problemas se } \\
\text { considera un aspecto central. }\end{array}$ \\
\hline \multirow{3}{*}{$\begin{array}{l}\text { Lugar de la } \\
\text { resolución de } \\
\text { problemas en e } \\
\text { desarrollo de } \\
\text { las unidades }\end{array}$} & $\begin{array}{l}\text { Resolución de problemas al } \\
\text { inicio de las unidades }\end{array}$ & $\begin{array}{c}\text { La resolución de problemas se considera al } \\
\text { inicio de los temas, como forma de incorporar } \\
\text { nuevos contenidos o habilidades. }\end{array}$ \\
\hline & $\begin{array}{l}\text { Resolución de problemas } \\
\text { durante el desarrollo de la } \\
\text { unidad }\end{array}$ & $\begin{array}{l}\text { La resolución de problemas se considera } \\
\text { durante el desarrollo de los temas. }\end{array}$ \\
\hline & $\begin{array}{c}\text { Resolución de problemas al } \\
\text { final de las unidades }\end{array}$ & $\begin{array}{c}\text { La resolución de problemas aparece al final de } \\
\text { los temas tratados. }\end{array}$ \\
\hline \multirow{7}{*}{$\begin{array}{l}\text { Entrega de } \\
\text { orientaciones } \\
\text { para los } \\
\text { profesores }\end{array}$} & Progreso de los estudiantes & $\begin{array}{c}\text { Se establecen especificaciones evidentes y } \\
\text { diferenciadas para cada nivel. }\end{array}$ \\
\hline & $\begin{array}{c}\text { Rol de la resolución de } \\
\text { problemas en el desarrollo de } \\
\text { un tema }\end{array}$ & $\begin{array}{c}\text { Se dan orientaciones sobre el objetivo que } \\
\text { cumple la resolución de problemas para el } \\
\text { desarrollo de los contenidos. }\end{array}$ \\
\hline & $\begin{array}{c}\text { Orientaciones sobre la } \\
\text { naturaleza de los problemas } \\
\text { matemáticos }\end{array}$ & $\begin{array}{c}\text { Se dan orientaciones sobre la naturaleza y } \\
\text { significancia de los problemas, su estructura y } \\
\text { propósito. }\end{array}$ \\
\hline & $\begin{array}{c}\text { Orientaciones sobre la } \\
\text { resolución de problemas } \\
\text { matemáticos }\end{array}$ & $\begin{array}{l}\text { Se dan orientaciones sobre modelos de } \\
\text { resolución y uso de estrategias heurísticas. }\end{array}$ \\
\hline & Invención de problemas & $\begin{array}{c}\text { Se dan orientaciones sobre características y } \\
\text { tipos de actividades de invención de problemas. }\end{array}$ \\
\hline & $\begin{array}{l}\text { Formas de pensar la } \\
\text { resolución de problemas }\end{array}$ & $\begin{array}{c}\text { Se dan orientaciones acerca de posibles formas } \\
\text { de pensamiento de los estudiantes al resolver un } \\
\text { problema (dificultades, conductas, etc.) }\end{array}$ \\
\hline & $\begin{array}{l}\text { Formas de trabajar la } \\
\text { resolución de problemas }\end{array}$ & $\begin{array}{l}\text { Se dan orientaciones sobre posibles enfoques } \\
\text { para trabajar la resolución de problemas, } \\
\text { estrategias metodológicas y metacognición del } \\
\text { estudiante. }\end{array}$ \\
\hline
\end{tabular}

La Dimensión 3 permite analizar los tipos de problemas sugeridos en los materiales curriculares. Para esto nos basamos en la clasificación de Zhu y Fan (2006), complementadas con aportes de Castro (1994) y la clasificación del tipo de situaciones de PISA (OECD, 2013). La Tabla 4 recoge las categorías y sub-categorías resultantes. 
Tabla 4. Dimensión 3: Tipos de problemas

\begin{tabular}{|c|c|c|}
\hline Categoría & Sub-categoría & Descripción \\
\hline \multirow{2}{*}{$\begin{array}{l}\text { Tipos de problemas } \\
\text { según conocimiento de } \\
\text { método de solución }\end{array}$} & Rutinarios & $\begin{array}{c}\text { La solución surge de aplicar algoritmo, } \\
\text { fórmula o procedimiento conocido. }\end{array}$ \\
\hline & No rutinarios & $\begin{array}{l}\text { La estrategia de solución no es } \\
\text { evidente. }\end{array}$ \\
\hline \multirow{2}{*}{$\begin{array}{l}\text { Tipos de problemas } \\
\text { según estrategia } \\
\text { metodológica }\end{array}$} & Tradicionales & $\begin{array}{c}\text { Son los que suelen aparecer en los } \\
\text { libros de texto. }\end{array}$ \\
\hline & No tradicionales & $\begin{array}{c}\text { Inventar problemas, problemas tipo } \\
\text { puzle, proyectos, diario matemático... }\end{array}$ \\
\hline \multirow{2}{*}{$\begin{array}{l}\text { Tipos de problemas } \\
\text { según cantidad de } \\
\text { respuestas posibles }\end{array}$} & De final cerrado & Una sola respuesta correcta. \\
\hline & De final abierto & Pueden tener varias posibles respuestas. \\
\hline \multirow{4}{*}{$\begin{array}{c}\text { Tipos de problemas } \\
\text { según tipo de situación } \\
\text { que aborda }\end{array}$} & Sobre situaciones personales & $\begin{array}{l}\text { Relacionados con el contexto inmediato } \\
\text { de los alumnos. }\end{array}$ \\
\hline & $\begin{array}{c}\text { Sobre situaciones educativas } \\
\text { o laborales }\end{array}$ & $\begin{array}{l}\text { Relacionados con la escuela o un } \\
\text { entorno de trabajo. }\end{array}$ \\
\hline & Sobre situaciones públicas & $\begin{array}{c}\text { Relacionados con la comunidad o } \\
\text { situaciones públicas. }\end{array}$ \\
\hline & Sobre situaciones científicas & $\begin{array}{l}\text { Implican el análisis de procesos } \\
\text { tecnológicos o situaciones } \\
\text { específicamente matemáticas. }\end{array}$ \\
\hline \multirow{2}{*}{$\begin{array}{c}\text { Tipos de problemas } \\
\text { según cantidad de } \\
\text { operaciones necesarias } \\
\text { para resolverlos }\end{array}$} & Simples & $\begin{array}{c}\text { Se necesita una sola clase de operación. } \\
\text { Implican solo dos datos numéricos. }\end{array}$ \\
\hline & Compuestos & $\begin{array}{l}\text { Se necesitan al menos dos operaciones } \\
\text { distintas, o una misma varias veces. }\end{array}$ \\
\hline \multirow{3}{*}{$\begin{array}{l}\text { Tipos de problemas } \\
\text { según suficiencia de } \\
\text { datos }\end{array}$} & Con datos suficientes & $\begin{array}{l}\text { Contienen exactamente los datos } \\
\text { suficientes para ser resuelto. }\end{array}$ \\
\hline & Con datos superfluos & Contienen más datos de los necesarios. \\
\hline & Con datos insuficientes & $\begin{array}{l}\text { El problema no contiene los datos } \\
\text { suficientes para resolverlo. }\end{array}$ \\
\hline \multirow{5}{*}{$\begin{array}{l}\text { Tipos de problemas } \\
\text { según tópico }\end{array}$} & Aritméticos & Abordan contenidos aritméticos. \\
\hline & Algebraicos & Abordan contenidos de álgebra. \\
\hline & Geométricos & Abordan contenidos de geometría. \\
\hline & De medición & Abordan contenidos de medición. \\
\hline & De estadística y probabilidad & $\begin{array}{l}\text { Abordan contenidos estadísticos y de } \\
\text { probabilidad. }\end{array}$ \\
\hline
\end{tabular}

Tras definir el sistema de categorías, identificamos en los documentos las unidades de análisis. Para las Dimensiones 1 y 2, las unidades fueron párrafos que incluyeran expresiones como "resolver problemas", "resuelven problemas" o "situaciones problemas". Para la Dimensión 3, las unidades fueron los ejemplos de problemas sugeridos en los documentos. A continuación, se realizó el proceso de codificación. En etapas tempranas de la investigación, se pidió a los dos colaboradores que aplicaran la codificación a partes de un mismo material. El grado de acuerdo resultó discreto, lo que llevó a describir en mayor profundidad algunas categorías e incorporar ejemplos de cada 
una. En una etapa avanzada se dio entrenamiento a uno de los colaboradores y se le pidió que codificara una parte más extensa del material. Revisamos el grado de acuerdo entre su codificación y la codificación de la primera autora, esta vez resultando alto.

\section{Resultados}

En esta sección, damos a conocer los resultados del análisis, organizados según los tres objetivos específicos de la investigación.

\subsection{Roles de la resolución de problemas en los documentos curriculares}

En cuanto a la Dimensión 1, a grandes rasgos, ninguno de los tres roles de Schroeder y Lester (1989) tuvo predominio exclusivo en los documentos. La Tabla 5 presenta un recuento de las veces que identificamos cada rol en los correspondientes documentos.

Tabla 5. Presencia de los tres roles en los documentos curriculares

\begin{tabular}{lllllllll}
\hline \multicolumn{1}{c}{ Roles } & DC1 & DC2 & DC3 & DC4 & DC5 & DC6 & DC7 & Total \\
\hline Enseñar para & 2 & 10 & 24 & 20 & 1 & 12 & 15 & 84 \\
Enseñar sobre & 0 & 12 & 51 & 10 & 5 & 6 & 5 & 89 \\
Enseñar a través & 0 & 14 & 30 & 17 & 7 & 1 & 17 & 88 \\
Total & 2 & 36 & 105 & 47 & 13 & 19 & 37 & 261 \\
\hline
\end{tabular}

La Ley que regula el sistema educativo (DC1) pone énfasis en la adquisición de conceptos y habilidades básicas. La resolución de problemas se presenta como un contexto para ponerlos en práctica, manifestándose el rol 'enseñar para resolver problemas'. De esta ley se derivan las Bases Curriculares (DC2). En ellas, la resolución de problemas se presenta como una de las cuatro habilidades que, junto con cinco ejes de contenido, estructuran los objetivos de aprendizaje de la educación primaria, manifestándose el rol 'enseñar sobre la resolución de problemas'. También encontramos evidencia de los otros dos roles. Por ejemplo, la siguiente cita corresponde a una unidad de análisis de la sub-categoría 'enseñar a través de la resolución de problemas':

La resolución de problemas es tanto un medio como un fin para lograr una buena educación matemática. Se habla de resolver problemas, en lugar de simples ejercicios, cuando el estudiante logra solucionar una situación problemática dada, contextualizada o no, sin que se le haya indicado un procedimiento a seguir. (MINEDUC, 2012a, p. 89)

Encontramos la enseñanza 'para resolver problemas' reflejada en las secuencias de objetivos de aprendizaje, donde dominan los procedimientos de cálculo aritmético antes que el uso de problemas para su ejercitación.

En el Programa de Estudio (DC3), se fomenta principalmente la enseñanza 'sobre' la resolución de problemas. Esto es debido a que en su estructura está considerado el presentar ejemplos de actividades, haciendo explícito las habilidades que se trabajan. En el caso de la Figura 1, además se observa la enseñanza 'para' resolver problemas, ya que estos aparecen tras haber aprendido el algoritmo necesario para resolverlos. Llama la atención que, de 27 secuencias de actividades propuestas para trabajar cada objetivo, solo cuatro inicien con algún tipo de tarea relativa a resolver (o inventar) problemas.

\begin{tabular}{|ll|}
\hline Actividades 2 y 3 & Resuelven problemas simples que involucren divisiones, usando \\
RESOLVER PROBLEMAS & el algoritmo de la actividad anterior: \\
Transferir los procedimientos & $>$ Se debe repartir 63 alumnos del $4^{\circ}$ nivel en 3 cursos con igual \\
utilizados en situacionesya & número de alumnos. \\
resueltas a problemas. (OA c) & $>$ Con un alambre de $84 \mathrm{~cm}$, se debe formar un cuadrado. \\
\hline
\end{tabular}

Figura 1: Actividad para el objetivo de aprendizaje 6 (MINEDUC, 2012b) 
En cambio, en el Material Pedagógico para el Aula (DC4), gran parte de las lecciones comienzan con situaciones problemáticas a partir de las cuales los alumnos construyen conocimiento. Un ejemplo se observa en la Figura 2, donde se espera que los estudiantes expliquen, comparen y evalúen sus estrategias de cálculo mental.

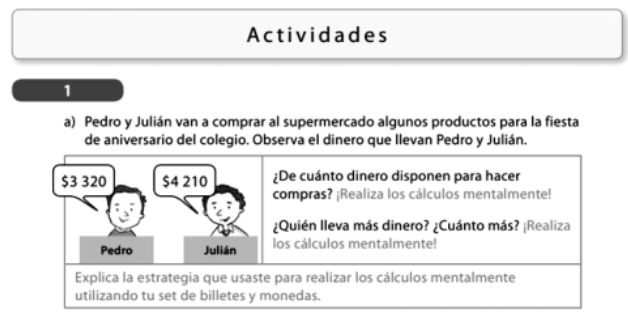

Figura 2. Actividad de inicio de la clase 5 del Material Pedagógico (MINEDUC, 2012c)

Otro documento analizado fue el que da las indicaciones para licitar los textos escolares que se entregan a todo el país (DC5). En él predomina el rol enseñar 'a través' de la resolución de problemas. Sin embargo, de un total de 51 criterios para la elaboración de los textos, sólo siete hacen referencia a la resolución de problemas.

La visión que transmite el Material Pedagógico (DC4) o las bases de licitación de textos no sigue la misma línea de lo requerido por las evaluaciones chilenas. En el documento que interpreta los resultados de los estudiantes en la prueba SIMCE según niveles de logro (DC6), predomina ampliamente la enseñanza de contenidos 'para' resolver problemas. Casi no se hace referencia a los otros dos roles y ni siquiera se alude a problemas en algunos ejes de contenidos.

Por último, en los estándares para la formación de profesores (DC7) se da énfasis a que los futuros docentes apliquen la resolución de problemas como metodología de enseñanza, y a que ellos mismos sepan resolver problemas de cada eje de contenido.

\subsection{Características metodológicas de la resolución de problemas}

Seguimos con resultados del análisis de la Dimensión 2 en los documentos, según categorías que desglosan características metodológicas de la resolución de problemas.

Evidencias de imbricación en el currículo. A primera vista los resultados reflejan la finalidad de cada documento: los Estándares de Aprendizaje (DC6) manifiestan énfasis en la evaluación. En los estándares para la formación de profesores (DC7) hay evidencia de imbricación en la enseñanza. Sin embargo, el Programa de Estudio (DC3), relacionado con la metodología, hace poca referencia a la resolución de problemas como método de enseñanza. Por el contrario, en el tipo de documento en donde hay evidencia de imbricación en el aprendizaje, la enseñanza y la evaluación, de forma más equilibrada fue en el Material Pedagógico para el Aula (DC4).

Tabla 6. Recuento para 'Lugar de la resolución de problemas en el desarrollo de las unidades'

\begin{tabular}{llll}
\hline \multicolumn{1}{c}{ Sub-categoría } & DC3 & DC4 & Total \\
\hline Resolución de problemas al inicio de las unidades & 6 & 23 & 29 \\
Resolución de problemas durante el desarrollo de las unidades & 13 & 30 & 43 \\
Resolución de problemas al final de las unidades & 15 & 8 & 23 \\
Total & 34 & 61 & 95 \\
\hline
\end{tabular}

Lugar de los problemas en el desarrollo de un tema. En dos de los materiales encontramos secuencias de actividades que involucraran problemas: Programa de Estudio (DC3) y Material Pedagógico (DC4). La Tabla 6 muestra un recuento de las unidades de análisis identificadas de acuerdo a esta sub-categoría. En las secuencias del 
Programa de Estudio (DC3) el lugar de los problemas corresponde, en su mayoría, al final de los temas tratados. Hay pocos problemas al inicio, excepto en los objetivos de aprendizaje directamente relacionados con resolver problemas. En las guías del Material Pedagógico (DC4), la mayoría de las secuencias comienzan con el planteamiento de situaciones problemas de las que surge el contenido matemático a trabajar.

Entrega de orientaciones para los profesores. Encontramos orientaciones dirigidas a los profesores en seis de los documentos curriculares. La Tabla 7 muestra un recuento de las unidades de análisis. En general, en la mayoría de las orientaciones se explica cómo trabajar los problemas propuestos y se proporcionan estrategias metodológicas para abordarlos. También se aborda la naturaleza de los problemas. En el Programa de Estudio (DC3) la mayoría de estas veces se explica la diferencia entre problemas rutinarios y no rutinarios. Encontramos pocas explicaciones sobre la invención de problemas, a pesar de ser una actividad fundamental ligada a la resolución de problemas.

Tabla 7. Recuento para 'Entrega de orientaciones para los profesores'

\begin{tabular}{|c|c|c|c|c|c|c|c|}
\hline Sub-categoría & $\mathrm{DC} 2$ & DC3 & DC4 & DC5 & DC6 & DC7 & Total \\
\hline Progreso de los estudiantes & 0 & 2 & 0 & 0 & 0 & 0 & 2 \\
\hline $\begin{array}{l}\text { Rol de la resolución de problemas en el } \\
\text { desarrollo de un tema }\end{array}$ & 0 & 0 & 10 & 0 & 0 & 0 & 10 \\
\hline $\begin{array}{l}\text { Orientaciones sobre la naturaleza de los } \\
\text { problemas matemáticos }\end{array}$ & 2 & 11 & 38 & 6 & 4 & 1 & 62 \\
\hline $\begin{array}{l}\text { Orientaciones sobre la resolución de problemas } \\
\text { matemáticos }\end{array}$ & 3 & 3 & 16 & 0 & 2 & 1 & 25 \\
\hline Invención de problemas & 1 & 1 & 6 & 1 & 0 & 0 & 9 \\
\hline Formas de pensar la resolución de problemas & 2 & 11 & 33 & 0 & 1 & 0 & 47 \\
\hline Formas de trabajar la resolución de problemas & 7 & 16 & 75 & 1 & 0 & 9 & 108 \\
\hline Total & 15 & 44 & 178 & 8 & 7 & 11 & 263 \\
\hline
\end{tabular}

\subsection{Tipos de problemas sugeridos para su implementación en el aula}

Los resultados de la Tabla 8 son para la Dimensión 3, referida a los tipos de problemas matemáticos. Hay problemas en tres de los documentos: Programa de Estudio (DC3), Estándares de Aprendizaje (DC6) y Material Pedagógico para el Aula (DC4).

Tabla 8. Recuento de unidades de análisis de la Dimensión 3: Tipos de problemas

\begin{tabular}{llllll}
\hline \multicolumn{1}{c}{ Dimensión: Tipo de problemas } & Categoría & D3 & D4 & D6 & Total \\
\hline \multirow{2}{*}{ Conocimiento de método de solución } & Rutinarios & 52 & 82 & 6 & 140 \\
& No rutinarios & 46 & 44 & 5 & 95 \\
\cline { 2 - 6 } Estrategia metodológica & Tradicionales & 91 & 115 & 11 & 217 \\
& No tradicionales & 7 & 9 & 0 & 16 \\
\cline { 2 - 6 } Cantidad de respuestas posibles & Cerrados & 84 & 111 & 10 & 205 \\
& Abiertos & 14 & 14 & 1 & 29 \\
\cline { 2 - 6 } Tipo de situación & Personales & 18 & 51 & 6 & 75 \\
& Educativas o laborales & 17 & 19 & 0 & 36 \\
& Públicas & 15 & 35 & 1 & 51 \\
Cantidad de operaciones & Científicas y matemáticas & 48 & 20 & 4 & 72 \\
\cline { 2 - 6 } Suficiencia de datos & Simples & 40 & 72 & 5 & 117 \\
& Compuestos & 57 & 53 & 6 & 116 \\
\cline { 2 - 6 } & Datos suficientes & 87 & 105 & 8 & 200 \\
& Datos superfluos & 9 & 17 & 3 & 29 \\
Tema que abordan & Datos insuficientes & 0 & 2 & 0 & 2 \\
\cline { 2 - 6 } & Aritméticos & 43 & 76 & 6 & 125 \\
& Algebraicos & 14 & 6 & 1 & 21 \\
& Geométricos & 17 & 18 & 2 & 37 \\
\hline
\end{tabular}




\begin{tabular}{lllll} 
De medición & 24 & 24 & 0 & 48 \\
De estadística y probabilidad & 0 & 1 & 2 & 3 \\
\hline
\end{tabular}

En el Programa de Estudio (DC3) hay un total de 98 problemas. Destaca que casi todos sean de tipo tradicional, es decir, que se pueden encontrar en los libros de texto. Además, predominan los problemas de tipo cerrado y sobre situaciones científicas o personales. Encontramos que, casi la mitad de los problemas es de tipo simple y, un poco más de la mitad, son de tipo compuesto. Además, casi la totalidad de problemas incluye la cantidad exacta de datos para ser resueltos. La mayoría de los problemas son aritméticos (43), seguidos por problemas de medición (24), geométricos (17) y algebraicos (14). No encontramos problemas sobre estadística o probabilidad.

En el Material Pedagógico (DC4) hay 126 problemas. De estos, la mayoría resultaron ser ejercicios de aplicación (82), pero a diferencia del Programa de Estudio (DC3), en casi todas las unidades hallamos problemas no rutinarios propuestos al inicio de las clases, aunque de tipo tradicional y cerrados. Predominan las situaciones personales (51). Los problemas simples (72) aparecen más que los compuestos (53). Hay más problemas con la cantidad exacta de información (105) y luego una pequeña cantidad de problemas con más datos de los necesarios (17). Este fue el único tipo de material donde encontramos dos problemas con datos insuficientes para ser resueltos; son de tipo acertijo que se sugiere proponer a los estudiantes al finalizar una evaluación.

En los Estándares de Aprendizaje (DC6), hallamos 11 ejemplos de problemas de la prueba SIMCE. Todos los problemas resultaron tradicionales e identificamos un solo problema abierto. Acerca del tipo de situaciones abordadas, la mayoría fueron de tipo personal. Casi la mitad de los problemas fueron simples (5) y la otra mitad compuestos (6). Predominan los problemas con la cantidad de datos justa para ser resueltos. Existe una mayor cantidad de problemas aritméticos (6) y no hay problemas de medición.

\section{Discusión y conclusiones}

Al caracterizar la presencia de la resolución de problemas en los documentos, obtuvimos resultados diversos. No siempre lo que se propone en las normativas curriculares guarda coherencia con la visión expuesta en el resto de los documentos. Por ejemplo, en las Bases Curriculares (DC2) se promueve la enseñanza a través de problemas, lo cual no coincide con la visión del Programa de Estudio (DC3), más enfocada en el desarrollo de la habilidad, ni con la cantidad de problemas rutinarios, tradicionales y cerrados que contiene. El Material Pedagógico (DC4) es el más cercano a Schoenfeld (1992) con los problemas como preguntas desconcertantes y la resolución de problemas como método de enseñanza del contenido habitual. Los Estándares de Aprendizaje (DC6) es uno de los materiales menos coherentes con la visión en las Bases Curriculares (DC2) al promover problemas rutinarios como tareas de ejercitación.

En cuanto a otras características de la resolución de problemas, cada documento demuestra evidencias de imbricación en el aprendizaje, la enseñanza o la evaluación de acuerdo a la finalidad que cumple. Acerca del lugar de los problemas en el tratamiento de los temas, los resultados son distintos entre el Programa de Estudio (DC3) y el Material Pedagógico (DC4). Este último presenta ejemplos interesantes del uso de problemas no rutinarios al inicio de secuencias didácticas. El resto de sub-categorías está presente de forma dispar en cada documento. La mayoría coincide en entregar mayor cantidad de orientaciones sobre la naturaleza de los problemas y cómo trabajarlos con los estudiantes. En cambio, hay pocas referencias a la invención de problemas. 
Acerca del tipo de problemas que aparecen en los documentos curriculares, la mayoría coinciden en promover los de tipo rutinario, tradicionales y de final cerrado, que tratan de situaciones personales, tienen la cantidad de datos exacta para ser resueltos y centrados en contenidos aritméticos.

Comparando nuestros resultados con otras investigaciones que analizan el currículo, el currículo chileno está lejos de ser similar, por ejemplo, al de Singapur, donde la resolución de problemas corresponde al elemento central que organiza el resto del currículo (Toh et al., 2019), o al de Japón, caracterizado por el enfoque de resolución estructurada de problemas (Fujii, 2018; Isoda, 2015). Los hallazgos concuerdan con Leong et al. (2016): a pesar de que la normativa declare la importancia de la resolución de problemas, los profesores suelen implementar una visión limitada. Semejante al caso analizado, donde la importancia que se da a la prueba SIMCE condiciona la forma de enseñar a resolver ciertos tipos de problemas, dando más relevancia a la transmisión de contenidos y procedimientos específicos antes que al desarrollo del pensamiento y habilidades matemáticas. Los resultados también son similares a los de López, Guerrero, Carrillo y Contreras (2015), quienes encuentran incongruencia entre lo exigido por el currículo (en este caso de secundaria) de España, orientado a la investigación y el tipo de problemas en textos escolares (ejercicios de repetición de fórmulas).

Nuestros resultados implican que los documentos curriculares, diseñados para apoyar la implementación de los objetivos de aprendizaje, carecen de una visión común sobre el papel de la resolución de problemas en la enseñanza de las matemáticas. Tampoco contribuyen con recursos de calidad, diversos y matemáticamente ricos para el uso por parte de los profesores, ya que por sobre todo el interés está puesto en el aprendizaje de contenidos y procedimientos evaluados por la prueba SIMCE. Como consecuencia, la actuación de los profesores se puede ver limitada por lo dispuesto en las normas curriculares, con pocas oportunidades para implementar metodologías que apunten al desarrollo del razonamiento a través de la búsqueda de soluciones. Por otro lado, queda de manifiesto una vez más lo difícil que resulta llevar a la práctica los avances de la didáctica de la matemática, dados los múltiples niveles de concreción por los que atraviesa el currículo, la cantidad de decisiones y de intereses que influyen hasta que los objetivos se transforman en resultados de aprendizaje.

En cuanto a las implicancias teóricas, este trabajo contribuye a integrar aspectos de la resolución de problemas que suelen ser investigados de manera independiente. Hemos visto que los tipos de problemas incluidos en materiales de apoyo a la instrucción contribuyen a reafirmar un cierto rol sobre la resolución de problemas. Así, los problemas rutinarios, cerrados y tradicionales colaboran a que se comprenda el uso de los problemas como forma de ejercitar contenidos enseñados por transmisión directa, de modo que se cumple el rol 'enseñar para resolver problemas'. Además, el análisis de aspectos como la entrega de sugerencias metodológicas, ha dado una visión más integral sobre cómo se aborda la resolución de problemas en este tipo de documentos, que luego se traducen en actividades de aula. Como trabajo futuro, conviene estudiar otros niveles de concreción curricular, tales como el currículo planificado por los profesores, el currículo enseñado o el currículo evaluado, poniendo a prueba e integrando los múltiples conocimientos sobre resolución de problemas con los que hoy en día se cuenta.

\section{Agradecimientos}

PGC2018-95765-B-I00, Ministerio de Ciencia, Innovación y Universidades. Beca CONICYT PFCHA/DOCTORADO BECAS CHILE/2018 folio 72190671. 


\section{Referencias}

Anderson, J. (2014). Forging new opportunities for problem solving in Australian mathematics classrooms through the first National Mathematics Curriculum. En Y. Li y G. Lappan (Eds.), Mathematics curriculum in school education (pp. 209-229). Dordrecht, Holanda: Springer.

Burkhardt, H. (2014). Curriculum design and systemic change. En Y. Li y G. Lappan (Eds.), Mathematics curriculum in school education (pp. 13-34). Dordrecht, Holanda: Springer.

Castro, E. (1994). Niveles de comprensión en problemas verbales de comparación multiplicativa. Trabajo de Tesis Doctoral. Universidad de Granada.

Castro, E. (2008). Resolución de problemas ideas, tendencias e influencias en España. En R. Luengo, B. Gómez, M. Camacho y L. Blanco (Eds.), Investigación en educación matemática XII (pp. 113-140). Badajoz: SEIEM.

Choppin, J., McDuffie, R., Drake, C. y Davis, J. (2018). Curriculum ergonomics: Conceptualizing the interactions between curriculum design and use. International Journal of Educational Research, 92, 75-85.

Fujii, T. (2018). Lesson study and teaching mathematics through problem solving: The two wheels of a cart. En M. Quaresma y otros (Eds.), Mathematics lesson study around the world. Theoretical and methodological issues (pp. 1-21). Cham, Suiza: Springer.

Isoda, M. (2015). The science of lesson study in the problem solving approach. En M. Inprasitha, M. Isoda, P. Wang-Iverson y B.-H. Yeap (Eds.), Lesson study: Challenges in mathematics education (Vol. 3, pp. 81-108). Singapur: World Sc.

Leong, Y., Tay, E., Toh, T., Quek, K., Toh, P. y Dindyal, J. (2016). Infusing mathematical problem solving in the mathematics curriculum: Replacement units. En P. Felmer, E. Pehkonen y J. Kilpatrick (Eds.), Posing and solving mathematical problems (pp. 309-325). Cham, Suiza: Springer.

Lester, F. y Cai, J. (2016). Can mathematical problem solving be taught? Preliminary answers from 30 years of research. En P. Felmer, E. Pehkonen y J. Kilpatrick (Eds.), Posing and solving mathematical problems (pp. 117-135). Cham, Suiza: Springer.

Ley $N^{\circ}$ 20.370. Ley General de Educación (2009). Biblioteca del Congreso Nacional de Chile. Recuperado 5/01/2018 de http://www.leychile.cl

López, E., Guerrero, A., Carrillo, J. y Contreras, L. (2015). La resolución de problemas en los libros de texto: un instrumento para su análisis. Avances de Investigación en Educación Matemática, 8, 74-94.

MINEDUC (2011). Estándares Orientadores para Egresados de Carreras de Pedagogía en Educación Básica. Recuperado 11/01/2018 de http://www.cpeip.cl/ estandares-orientadores-para-la-formacion-inicial-docente

MINEDUC (2012a). Bases Curriculares para la Educación Básica. Recuperado 12/01/2018 de http://archivos.agenciaeducacion.cl/biblioteca_digital_historica/ orientacion/2012/bases_curricularesbasica_2012.pdf

MINEDUC (2012b). Material Pedagógico Matemáticas. Recuperado 21/01/2018 de https://basica.mineduc.cl/matematica 
MINEDUC (2012c). Programas de Estudio para la Educación Básica. Recuperado 05/01/2018 de http://www.curriculumnacional.cl/inicio/1b-6b

MINEDUC (2013). Estándares de Aprendizaje Matemáticas $4^{\circ}$ básico. Recuperado 10/01/2018 de http://www.curriculumnacional.cl/wp-content/uploads/1b_6b/otros/ Estándares de Aprendizaje Matemáticas_4o básico.pdf

MINEDUC (2017). Bases administrativas, bases técnicas y anexos de licitación pública para la adquisición de textos para los estudiantes, guías para los docentes y recursos digitales complementarios, destinados a estudiantes y profesores de educación básica y media. Santiago de Chile: Ministerio de Educación.

OECD (2013). Draft PISA 2015. Mathematics framework. París: OECD.

Oliva, M. (2017). Arquitectura de la política educativa chilena (1990-2014): El currículum, lugar de la metáfora. Revista Brasileira de Educação, 22(69), 405-428.

Piñeiro, J., Castro-Rodríguez, E. y Castro, E. (2016). Resultados PISA y resolución de problemas matemáticos en los currículos de Educación Primaria. Edma 0-6, 5(2), 50-64.

Remillard, J. y Heck, D. (2014). Conceptualizing the curriculum enactment process in mathematics education. ZDM-Mathematics Education, 46(5), 705-718.

Rico, L. y Moreno, A. (Eds.). (2016). Elementos de didáctica de la matemática para el profesor de secundaria. Madrid: Pirámide.

Ruminot, C. (2017). Los efectos adversos de una evaluación nacional sobre las prácticas de enseñanza de las matemáticas: El caso de SIMCE en Chile. Revista Iberoamericana de Evaluación Educativa, 10(1), 69-87.

Schoenfeld, A. (1985). Mathematical problem solving. Nueva York: Academic Press.

Schoenfeld, A. (1992). Learning to think mathematically: Problem solving, metacognition and sense of mathematics. En D. Grows (Ed.), Handbook of research on mathematics teaching and learning (pp. 334-370). Nueva York: Macmillan.

Schroeder, T. y Lester, F. (1989). Developing understanding in mathematics via problem solving. En P. Trafton y A. Shulte (Eds.), New directions for elementary school mathematics (pp. 31-42). Reston, VA: NCTM.

Stacey, K. (2005). The place of problem solving in contemporary mathematics curriculum documents. The Journal of Mathematical Behavior, 24(3-4), 341-350.

Toh, T., Chan, C., Tay, E. Leong, Y., Quek, K., Toh, P.... Dong, F. (2019). Problem solving in the Singapore school mathematics curriculum. En T. Toh, B. Kaur y G. Tay (Eds.), Mathematics education in Singapore (pp. 141-164). Singapur: Springer.

Zhu, Y. y Fan, L. (2006). Focus on the representation of problem types in intended curriculum: A comparison of selected mathematics textbooks from mainland China and the United States. International Journal of Science and Mathematics Education, $4,609-626$.

\section{Referencias de los autores}

Daniela Olivares, Universidad de Granada (España). danielaod@correo.ugr.es

Isidoro Segovia, Universidad de Granada (España). isegovia@ugr.es

José Luis Lupiáñez, Universidad de Granada (España). lupi@ugr.es 


\title{
Roles of problem solving in the official curriculum
}

\author{
Daniela Olivares, Universidad de Granada \\ Isidoro Segovia, Universidad de Granada \\ José Luis Lupiáñez, Universidad de Granada
}

Research in mathematics education has come a long way in the field of problem solving. This is nonetheless far from always reflected in classroom practice. Some authors point to reasons such as conflicting messages about the role of problem solving in different types of documents and curriculum materials (Burkhardt, 2014). In this article, we describe the roles of problem solving in Chilean official curricular documents. We ground on the model of the curriculum system by Remillard and Heck (2014), which defines the mathematics curriculum and indicates the components of the official curriculum: objectives, materials to support implementation, and contents of evaluation. We then consider the roles that can be given to problem solving according to Schoenfeld (1992) (as anything that requires doing something, or as a perplexing or difficult question) and Schroeder and Lester (1989) (teaching for, about or through problem solving). We also consider a set of literature-based characteristics that facilitate the incorporation of problem solving into the curriculum, as well as the types of problems that can be found in the supporting documents. We develop a system of categories based on our theoretical framework. By means of these categories, we conduct a content analysis procedure for the main Chilean curriculum documents: Official Regulations, Study Program, support materials, documents on the national SIMCE assessment, and standards for teacher training, among others. We focus the analysis on the 4th grade of primary education. The results show that different roles predominate throughout the curricular documents. While Official Regulations (DC2) promote teaching through problem solving, this is not equally reflected in the other documents. In addition, Support Materials (DC3) provide guidance on the nature of the problems, and on how to work with them, but they hardly refer to student progress or to problem posing. Finally, we find that the types of problems suggested do not always contribute to the vision regarding problem solving and mathematics teaching as declared. This is observable in the promotion of and reference to routine, closed and traditional problems. 